\title{
Comparing Cyclic Tension-Compression Effects on CoCrFeMnNi High-Entropy Alloy and Ni-Based Superalloy
}

\author{
Tu-Ngoc Lam ${ }^{1}$, You-Shiun Chou ${ }^{1}$, Yao-Jen Chang ${ }^{2,3}$, Tsung-Ruei Sui ${ }^{1}$, An-Chou Yeh ${ }^{2,3}$, \\ Stefanus Harjo ${ }^{4}$, Soo Yeol Lee ${ }^{5, *}$, Jayant Jain ${ }^{6}$, Bo-Hong Lai ${ }^{1}$ and E-Wen Huang ${ }^{1, *}$ \\ 1 Department of Materials Science and Engineering, National Chiao Tung University, Hsinchu 30010, Taiwan \\ 2 Department of Materials Science and Engineering, National Tsing Hua University, Hsinchu 30013, Taiwan \\ High Entropy Materials Center, National Tsing Hua University, Hsinchu 30013, Taiwan \\ 4 J-PARC Center, Japan Atomic Energy Agency, 2-4 Shirane Shirakata, Tokai-mura, Naka-gun, \\ Ibaraki 319-1195, Japan \\ 5 Department of Materials Science and Engineering, Chungnam National University, Daejeon 34134, Korea \\ 6 Department of Materials Science and Engineering, Indian Institute of Technology, New Delhi 110016, India \\ * Correspondence: sylee2012@cnu.ac.kr (S.Y.L.); EwenHUANG@nctu.edu.tw (E.-W.H.)
}

Received: 12 July 2019; Accepted: 8 August 2019; Published: 13 August 2019

check for updates

\begin{abstract}
An equal-molar CoCrFeMnNi, face-centered-cubic (fcc) high-entropy alloy (HEA) and a nickel-based superalloy are studied using in situ neutron diffraction experiments. With continuous measurements, the evolution of diffraction peaks is collected for microscopic lattice strain analyses. Cyclic hardening and softening are found in both metallic systems. However, as obtained from the diffraction-peak-width evolution, the underneath deformation mechanisms are quite different. The CoCrFeMnNi HEA exhibits distinct lattice strain and microstructure responses under tension-compression cyclic loadings.
\end{abstract}

Keywords: in-situ neutron diffraction; low-cycle fatigue; high entropy alloy; Ni-based superalloy

\section{Introduction}

Fracture toughness and fatigue behavior are the key to the practical applications of high entropy alloys (HEAs) [1]. Hemphill et al.'s [2] four-point-bending fatigue experiments suggest that the fatigue behavior of $\mathrm{Al}_{0.5} \mathrm{CoCrCuFeNi} \mathrm{HEA}$ is affected by microstructural defects of oxides and microcracks while Tang et al.'s [3] high-cycle four-point-bending fatigue experiments reveal that nanotwinning is the major deformation behavior before fracture in a $\mathrm{Al}_{0.5} \mathrm{CoCrCuFeNi}$ two-phase HEA. Despite the convenience of four-point-bending high-cycle fatigue (HCF), more detailed fatigue mechanisms, especially in the low-cycle fatigue (LCF) in HEAs, need to be further explored, since the deformation behaviors under HCF and LCF regimes are very different, as suggested by Chen et al.'s review [4].

Thurston et al. [5] report that fatigue behavior of the Cantor alloy is comparable to the austenitic stainless steels and twinning induced plasticity (TWIP) steels. Recently, Niendorf et al.'s [6] tension-compression LCF experiments indicate the influence of planarity of slip and partial reversibility of deformation on the unobvious cyclic hardening at given plastic strain in $\mathrm{Fe}_{50} \mathrm{Mn}_{30} \mathrm{Co}_{10} \mathrm{Cr}_{10}$ HEA. There are few reports revealing the polymorphism of $\mathrm{CoCrFeMnNi}$, which is induced by tension [7] and compression [8-10], respectively. Moreover, the cyclic tension-compression effects as well as predominant mechanisms during the LCF process in CoCrFeMnNi still remain elusive. Meanwhile, the cyclic-loading deformation in face-centered-cubic (fcc) nickel-based superalloy has been extensively examined in which lattice strain asymmetry associated with dislocation density evolution governs the irreversible plastic deformation [11-14]. We herein investigate the in-situ 
tension-compression cyclic loading effects and the dominant deformation behaviors of the CoCrFeMnNi HEA in comparison with the commercially popular nickel-based superalloy by exploiting in-situ neutron diffraction measurements.

\section{Materials and Methods}

The nickel-based superalloy HASTELLOY C22 $2^{\mathrm{TM}}$ (Ni-21Cr-17Mo in weight percentage) was annealed to dissolve most precipitates into the fcc matrix structure. $\mathrm{C} 22^{\mathrm{TM}}$ has been chosen as a reference because this $\mathrm{Ni}$-based superalloy has been extensively studied using neutron diffraction and other complimentary experiments [11-17]. Several correlations from diffraction-data characteristics, microscopic images and underneath deformation mechanisms have been found as benchmarks and references. Hence, we follow the same protocol and experimental design performed on the $\mathrm{C} 22^{\mathrm{TM}}$ to apply to the current CoCrFeMnNi HEA examinations. In addition, the LCF experiments and cyclic-loading deformation mechanisms of the $\mathrm{C} 22^{\mathrm{TM}}$ are clarified and cited in many articles [11-16].

For the CoCrFeMnNi HEA, the sample was fabricated by vacuum arc-melting (arc) with equal molar compositions of constituent powders whose purity is more than $99.9 \%$ (in weight percent) and the diffraction characteristics of the $\mathrm{CoCrFeMnNi} \mathrm{HEA} \mathrm{are} \mathrm{also} \mathrm{well} \mathrm{studied} \mathrm{for} \mathrm{its} \mathrm{microstructure} \mathrm{[10,18-20].}$

The selected gauge volume for both samples was approximately $120 \mathrm{~mm}^{3}$ to associate the microstructure with the bulk properties. During cyclic deformation of the $\mathrm{C} 22^{\mathrm{TM}}$ alloy, in situ neutron diffraction measurements with the Spallation Neutron Source were carried out at the Spectrometer for Materials Research at Temperature and Stress (SMARTS) beamline of Los Alamos Neutron Science Center (LANSCE). The in situ neutron diffraction measurements for LCF of the HEA were conducted at TAKUMI beamline in Materials and Life Science Experimental Facilities at J-PARC, Japan. The loading direction is $45^{\circ}$ relative to the incident neutron beam direction, with two detector banks located at $\pm 90^{\circ}$ concomitantly recording diffraction vector patterns in the loading and transverse directions. Both the in situ neutron diffraction LCF tests were conducted at room temperature with the maximum tensile strain of $1 \%$ and the maximum compressive strain of $-1 \%$. The cyclic frequency was $1 \mathrm{~Hz}$. The neutron diffraction profiles were analyzed by applying pseudo-Voigt function for single peak fitting with the General Structure Analysis Software (GSAS, Los Alamos National Laboratory, version 1, Los Alamos County, New Mexico, United States) [21] and Convolutional Multiple Whole Profile (CMWP) [22] software, following the previously used protocol $[13,15]$.

\section{Results}

\subsection{Chemical Composition of Alloying Elements in CoCrFeMnNi}

We employed JEOL 6500 scanning electron microscopy (SEM, Japan Electron Optics Laboratory, Tokyo, Japan) with energy-dispersive x-ray (EDX, Japan Electron Optics Laboratory, Tokyo, Japan) spectroscopy for determining the elemental composition. The EDX analysis in Figure 1 discloses the alloy composition of $\mathrm{CoCrFeMnNi}$ with an equal weight percentage distribution of each constituent element, determining the $\mathrm{Co}_{20} \mathrm{Cr}_{20} \mathrm{Fe}_{20} \mathrm{Mn}_{20} \mathrm{Ni}_{20}$ HEA in the present study. 


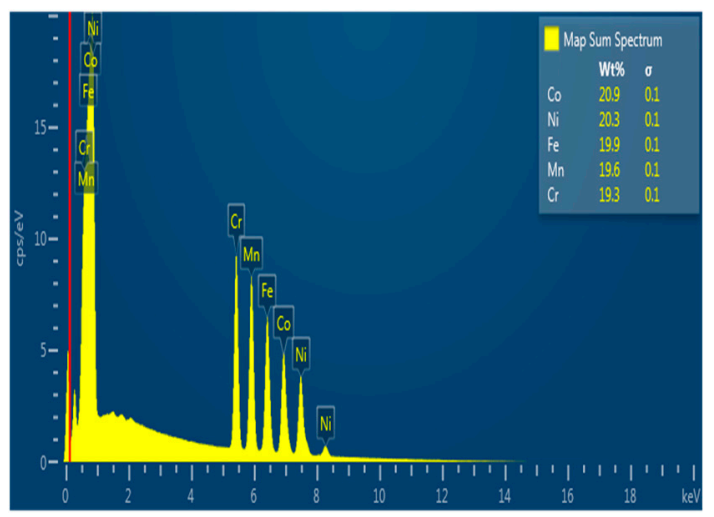

Figure 1. EDX spectrum of the CoCrFeMnNi HEA.

\subsection{Low-Cycle Fatigue Experiments}

Figure 2 presents the macroscopic stresses evolution versus certain selected fatigue cycles measured at the maximum strain of $1 \%$ and the minimum strain of $-1 \%$ for $C 22^{\mathrm{TM}}$ and $\mathrm{CoCrFeMnNi}$ alloys. Both the $\mathrm{C} 22^{\mathrm{TM}}$ and HEA performed similar cyclic hardening followed by softening tendency, however, the microscopic mechanisms underlying their cyclic hardening/softening-structural transformation were in different behaviors. Three distinct regions of the fatigue cycles based on the applied stress development can be clearly observed. During continuous tension-compression LCF deformation, the $\mathrm{C} 22^{\mathrm{TM}}$ alloy revealed the cyclic hardening within the 100th cycle region with increasing applied stress (stage I), followed by the cyclic softening upon the 1000th cycle with a gradual decrease in applied stress (stage II) and remaining saturated (stage III) until the 2500th cycle. Compared to the C22 ${ }^{\mathrm{TM}}$, the HEA experienced the hardening/softening transition at the 10th fatigue cycle, and then cyclic softening. At the same strain of tensile $(1 \%)$ or compressive $(-1 \%)$ deformation, an increase in stress up to the hardening/softening transition on the $\mathrm{C} 22^{\mathrm{TM}}$ was more pronounced than that on the HEA, which demonstrated the greater hardening behavior of the $\mathrm{C} 22^{\mathrm{TM}}$ alloy.

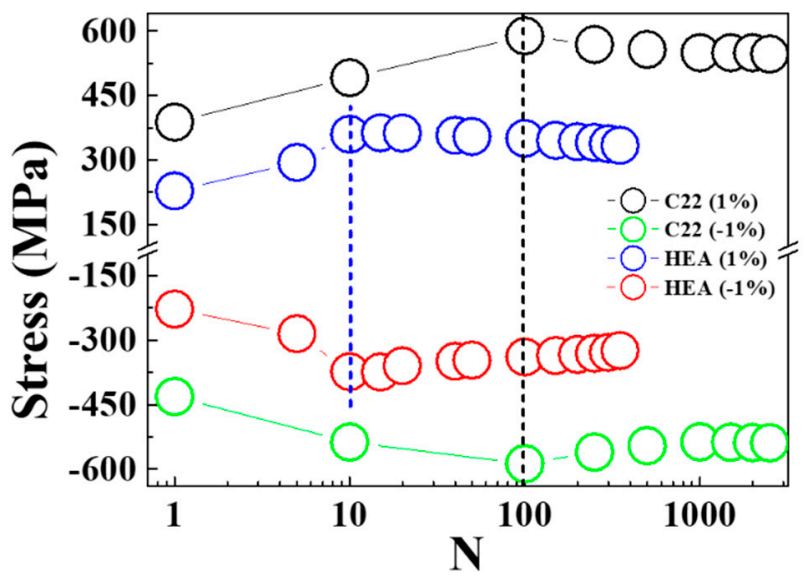

Figure 2. The stress evolution at the maximum tension (1\%) and compression $(-1 \%)$ as a function of fatigue cycle (presented in log scale) for both the $\mathrm{C}^{2} 2^{\mathrm{TM}}$ and $\mathrm{CoCrFeMnNi}$ alloys.

\subsection{Lattice Strain Evolution during Tension-Compression Cyclic Loadings}

The lattice strain evolution was calculated based on the variations in the d-spacing of the refined neutron diffraction peak positions of lattice planes as a function of fatigue cycle with respect to the initial d-spacing before deformation. Figure 3 depicts the lattice strain-stress dependence of the $\{311\}$ plane at the maximum tensile (1\%) and compressive $(-1 \%)$ strains in both transverse and axial loading directions since $\{311\}$ grain families are the least affected crystallographic planes by intrinsic microstructure deformation rather than external loadings in fcc polycrystalline materials 
under loading $[12,23]$. The yellow arrows show the trends of lattice strain evolution under cyclic loadings. Within the cyclic hardening stage in the early fatigue cycles, we can observe a positive correlation between the elastic strain and applied stress in both C22 ${ }^{\mathrm{TM}}$ and HEA, which seems to follow Hooke's law as Suzuki and Egami reported for cyclic deformation [24]. Specifically, C22 ${ }^{\mathrm{TM}}$ has a nearly linear relationship up to the hardening/softening transition. However, although the lattice strain-stress relationship of HEA is also positive up to the 10th fatigue cycle, the trend is not linear. Several reports suggest that such a nonlinear trend may be governed by the texture, patterned dislocation structure, or other anisotropy of the materials [11].

\section{(a)}

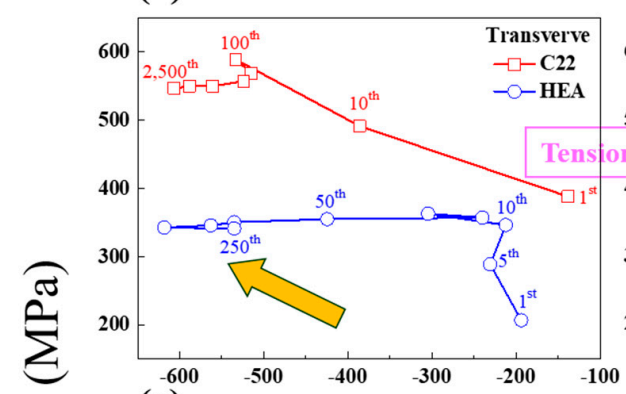

(c) (b)

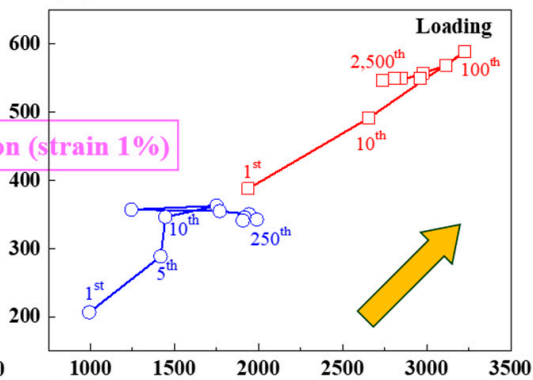

(d)
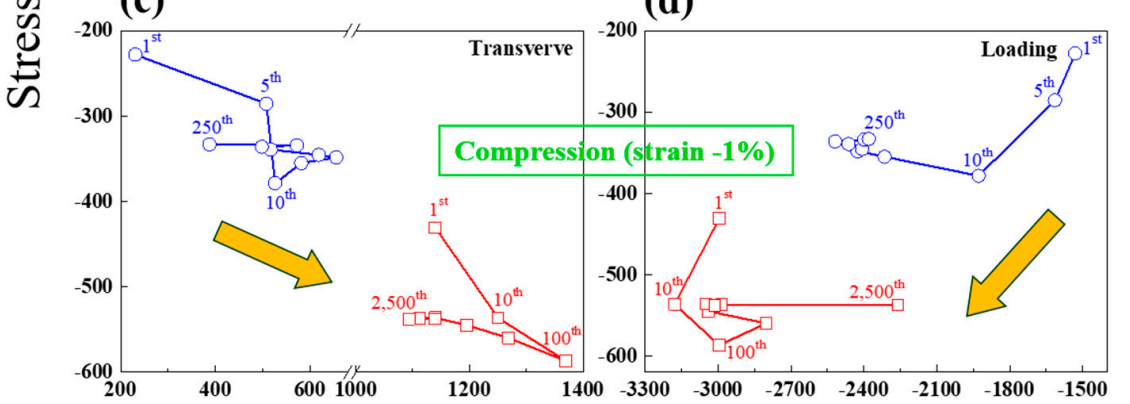

$\{311\}$ lattice strain $\left(10^{-6}\right)$

Figure 3. The $\{311\}$ lattice strain-stress relationship at the maximum tensile strain of $1 \%$ in (a) transverse and (b) axial loading; and at the maximum compressive strain of $-1 \%$ in (c) transverse and (d) axial loading directions for both the $\mathrm{C} 22^{\mathrm{TM}}$ and $\mathrm{CoCrFeMnNi}$ alloys.

In the hardening cycles of stage I, both the $\mathrm{C} 22^{\mathrm{TM}}$ and HEA displayed the same propensity in which the lattice strain increases with the number of fatigue cycles under both tensile and compressive deformation in both axial loading and transverse directions, ascribed to the cyclic hardening behavior in this region. However, the increase of elastic strain in the $\mathrm{C} 22^{\mathrm{TM}}$ was much greater than that of the HEA under maximum tension shown in Figure 3a,b. Meanwhile, the lattice strain evolution of the $\mathrm{C} 22^{\mathrm{TM}}$ and HEA was almost the same under maximum compressive deformation within the cycling hardening region. In stage II, there was an opposite trend of lattice strain evolution between the two alloys. The lattice strain of HEA continued to increase while that of $\mathrm{C} 22^{\mathrm{TM}}$ experienced an initially slight decrease within the cyclic softening region. Furthermore, the elastic strain of $\mathrm{C} 22^{\mathrm{TM}}$ increases under tension in Figure 3a but decreases under compression in Figure 3c from the 250th to the 1000th fatigue cycle for the grains oriented along the transverse direction. A similar tendency was also obtained for the grains of $\mathrm{C} 22^{\mathrm{TM}}$ oriented along the axial loading direction in tension in Figure $3 \mathrm{~b}$ and compression in Figure 3d. The deviation of elastic strain within the cyclic softening region was ascribed to the development of the intergranular strains [16]. In stage III, there was a gradually decrease of lattice strain of $\mathrm{C} 22^{\mathrm{TM}}$ under both tensile and compressive tests for grains oriented along both axial loading and transverse directions after the 1000th cycle. Similar to the $\mathrm{C} 22^{\mathrm{TM}}$ alloy, the lattice strain of HEA also depicted a slight decrease after the 200th cycle. The major discrepancy in the lattice strain evolution of 
the $\mathrm{C} 22^{\mathrm{TM}}$ and HEA was further clarified in terms of the dominant development of dislocation slip activities and other fatigue-induced defects such as stacking faults and twins.

\section{Discussion}

In this work, we determine the evolution of average dislocation density (n) and dislocation-wall spacings (D) by using CMWP software [22] in fitting the neutron diffraction profiles. Figure 4a depicts the dislocation density and dislocation wall spacings as a function of fatigue cycle at the maximum strain of $1 \%$ in both $\mathrm{C} 22^{\mathrm{TM}}$ [13] and $\mathrm{CoCrFeMnNi}$ alloys along the axial loading direction. For $\mathrm{CoCrFeMnNi}$, the dislocation density sharply increased together with an increasing number of fatigue cycles in the very early stages in the axial loading direction and reached the maximum value at the 40th cycle, then decreased with further fatigue cycles undergoing the softening cycles. The decrease in the density of the randomly distributed dislocations after the hardening cycles seen in the $\mathrm{CoCrFeMnNi}$ was analogous to that obtained in the $\mathrm{C}^{2} 2^{\mathrm{TM}}$ alloy [13]. The average distances between the dislocation-wall structures are largest at a very early cycle because the annealed C22 ${ }^{\mathrm{TM}}$ alloy has fewer cell structures than Mughrabi's dislocation wall [25] in the beginning [17]. The C22 ${ }^{\mathrm{TM}}$ alloy mainly has randomly distributed dislocations before cyclic loading [17]. Upon cyclic loading, the average distances between the dislocation-wall structures dropped within the hardening region, and remained constant with higher fatigue cycles undergoing the softening and saturation regimes. Due to dislocation movement and interaction, a more orderly arrangement of dislocations associated with the formation of the Mughrabi's dislocation-wall for cell structures resulted in a decrease and final saturation of the dislocation-wall distance when the dislocation densities approached a more stable state in $\mathrm{CoCrFeMnNi}$. The dislocation hardening was demonstrated to be the main deformation mechanism of the cyclic hardening effect in the $\mathrm{C} 22^{\mathrm{TM}}$ alloy. The calculated dislocation-wall-spacing evolution within the hardening region of HEA is similar to that of $\mathrm{C} 22^{\mathrm{TM}}$ but the dislocation density evolution of HEA is quite different from that of $\mathrm{C} 22^{\mathrm{TM}}$, implying that there are other microstructure formations coupled with cyclic deformation activities in governing the LCF regime. It has been reported that mechanical nanotwinning is the major deformation mechanism before fatigue crack initiation and propagation in HEA [3,4,26,27].

The generation of different kinds of defects associated with the dislocation density evolution under LCF was further elucidated. As reported by Ungar, each kind of defect is simultaneously governed by distinct diffraction peak aberrations such as peak shift, peak broadening, peak asymmetry, anisotropic peak broadening, and peak shape [28]. We first examined the deformation fault probability of $\mathrm{CoCrFeMnNi}$ obtained directly from the changes in only diffraction peak separations, which is applied only for fcc structure, as summarized by Warren [29]. Using this method, we can predict the presence of deformation fault probability based on only peak-shift correlations between (111) and (2 00 ) of polycrystalline metals in fcc structure, specifically, the displacement of $d_{111}-d_{200}$ separation toward smaller or larger deviation between these two groups of lattice planes, as shown in Figure $4 \mathrm{~b}$. However, we cannot distinguish whether the deformation fault is intrinsic or extrinsic stacking faults. To attain the quantitative probability of intrinsic, extrinsic stacking faults, and twinning, a more complete and reliable analysis of CMWP program [22] was further employed to resolve the convoluted diffraction data and the corresponding results are presented in Figure 4c. 
(a)

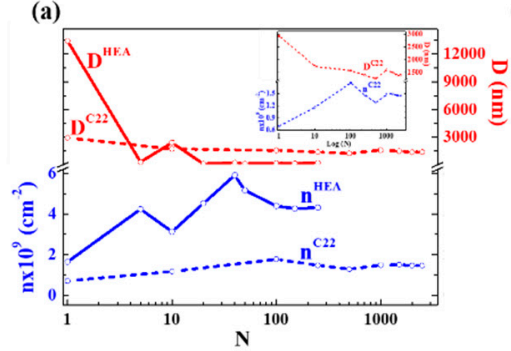

(b)

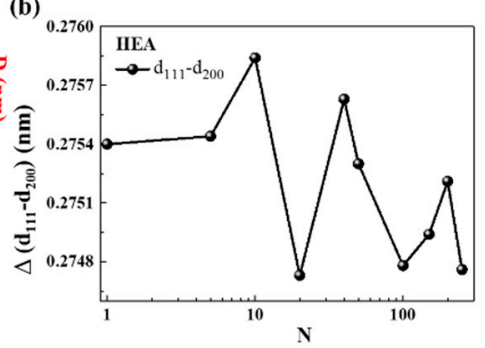

(c)

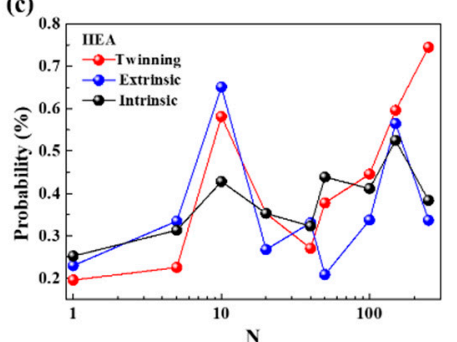

Figure 4. (a) The dislocation density and dislocation-wall-spacing evolution in $\mathrm{C} 22^{\mathrm{TM}}$ and $\mathrm{CoCrFeMnNi}$ alloys. The magnification of $\mathrm{n}$ and $\mathrm{D}$ of $\mathrm{C} 22^{\mathrm{TM}}$ alloy is indicated in the inset. (b) The difference in d-spacing between diffraction peaks 111 and 200 of CoCrFeMnNi. (c) Twining, extrinsic and intrinsic stacking faults defects as a function of fatigue cycle (presented in log scale) in CoCrFeMnNi.

It can be seen from CMWP fitting results in Figure 4c that the formation probability of twinning, extrinsic and intrinsic stacking faults defects started to slightly increase with the dislocation density evolution and the extrinsic stacking fault plays a major role in deformation in the hardening cycles. More twinning and extrinsic stacking faults were generated easily, and they governed the cyclic hardening behavior. Further increase of dislocation densities led to the decrease of twinning, and the probability of extrinsic and intrinsic stacking faults in the first half of softening cycles. When the dislocation densities were in the more stable condition, there was a slight variation of extrinsic and intrinsic stacking faults but an obvious increasing trend of twinning probability, suggesting the dominant role of twinning in the latter half of softening cycles. The formation of nanotwins and stacking faults is attributed to the low stacking fault energy (SFE) of HEA.

\section{Conclusions}

During low-cycle fatigue experiments, both the CoCrFeMnNi and C22 ${ }^{\mathrm{TM}}$ alloys display obvious cyclic hardening and softening regimes, however, the $\mathrm{CoCrFeMnNi}$ indicated fewer hardening cycles comparing to the $\mathrm{C} 22^{\mathrm{TM}}$ alloy. The lattice strain-stress relationship of these two alloys are quite different, indicating that dominant deformation-induced microstructures were distinct under tension-compression cyclic loadings. The twinning, extrinsic and intrinsic stacking faults are found to be formed during cyclic hardening regimes, and twinning is the most predominant defect in governing the main deformation behavior of $\mathrm{CoCrFeMnNi}$ in the latter half of softening regimes. The role of twin formation subjected to tension-compression cyclic loadings on CoCrFeMnNi is revealed, which may be useful for HEA design for fatigue endurance.

Author Contributions: Conceptualization, E.-W.H.; data analysis, T.-N.L., Y.-S.C., B.-H.L. and E.-W.H.; investigation, Y.-J.C. and T.-R.S.; project administration, E.-W.H.; resources, A.-C.Y. and S.H.; supervision, E.-W.H.; writing-original draft, T.-N.L. and E.-W.H.; writing-review and editing, A.C.Y., S.Y.L. and J.J.

Funding: The neutron diffraction experiments were performed at BL19 in Materials and Life Science Experimental Facility of J-PARC with the proposal 2016A0322. The authors are grateful to the support of the Ministry of Science and Technology (MOST) Programs, 107-2218-E-007-012, 107-3017-F-009-002, 107-3017-F-007-003, 107-3017-F-009-002, 107-3011-F-002-002, and 108-2221-E-009-131-MY4. This work was financially supported by the "Center for the Semiconductor Technology Research" from The Featured Areas Research Center Program within the framework of the Higher Education Sprout Project by the Ministry of Education (MOE) in Taiwan. Additionally supported in part by the MOST, Taiwan, under Grant MOST-108-3017-F-009-003. EWH and TNL are supported by MOST 107-2628-E-009-001-MY3. SYL was supported by a National Research Foundation (NRF) grant funded by the Korean government (No.2017K1A3A7A09016308).

Acknowledgments: We sincerely appreciate Tamás Ungár for his Convolutional Multiple Whole Profile (CMWP) software. EWH very much appreciate the financial support from the National Synchrotron Radiation Research Center (NSRRC) Neutron Program. This work has benefited from the use of Spectrometer for Materials Research at Temperature and Stress (SMARTS) of the Lujan Neutron Scattering Center at LANSCE, which is funded by the Department of Energy's Office of Basic Energy Sciences. Los Alamos National Laboratory is operated by Los Alamos National Security LLC under DOE.

Conflicts of Interest: The authors declare no conflicts of interest. 


\section{References}

1. Seifi, M.; Li, D.; Yong, Z.; Liaw, P.K.; Lewandowski, J.J. Fracture Toughness and Fatigue Crack Growth Behavior of As-Cast High-Entropy Alloys. Jom 2015, 67, 2288-2295. [CrossRef]

2. Hemphill, M.A.; Yuan, T.; Wang, G.Y.; Yeh, J.W.; Tsai, C.W.; Chuang, A.; Liaw, P.K. Fatigue behavior of A10.5CoCrCuFeNi high entropy alloys. Acta Materialia 2012, 60, 5723-5734. [CrossRef]

3. Tang, Z.; Yuan, T.; Tsai, C.W.; Yeh, J.W.; Lundin, C.D.; Liaw, P.K. Fatigue behavior of a wrought Al0.5CoCrCuFeNi two-phase high-entropy alloy. Acta Materialia 2015, 99, 247-258. [CrossRef]

4. Chen, P.; Lee, C.; Wang, S.Y.; Seifi, M.; Lewandowski, J.J.; Dahmen, K.A.; Jia, H.; Xie, X.; Chen, B.; Yeh, J.W.; et al. Fatigue behavior of high-entropy alloys: A review. Sci. China Technol. Sci. 2017, 61, 168-178. [CrossRef]

5. Thurston, K.V.S.; Gludovatz, B.; Hohenwarter, A.; Laplanche, G.; George, E.P.; Ritchie, R.O. Effect of temperature on the fatigue-crack growth behavior of the high-entropy alloy CrMnFeCoNi. Intermetallics 2017, 88, 65-72. [CrossRef]

6. Niendorf, T.; Wegener, T.; Li, Z.; Raabe, D. Unexpected cyclic stress-strain response of dual-phase high-entropy alloys induced by partial reversibility of deformation. Scr. Mater. 2018, 143, 63-67. [CrossRef]

7. Niu, C.; LaRosa, C.R.; Miao, J.; Mills, M.J.; Ghazisaeidi, M. Magnetically-driven phase transformation strengthening in high entropy alloys. Nat. Commun. 2018, 9, 1363. [CrossRef]

8. Tracy, C.L.; Park, S.; Rittman, D.R.; Zinkle, S.J.; Bei, H.; Lang, M.; Ewing, R.C.; Mao, W.L. High pressure synthesis of a hexagonal close-packed phase of the high-entropy alloy CrMnFeCoNi. Nat. Commun. 2017, 8, 15634. [CrossRef]

9. Zhang, F.; Wu, Y.; Lou, H.; Zeng, Z.; Prakapenka, V.B.; Greenberg, E.; Ren, Y.; Yan, J.; Okasinski, J.S.; Liu, X.; et al. Polymorphism in a high-entropy alloy. Nat. Commun. 2017, 8, 15687. [CrossRef]

10. Huang, E.W.; Lin, C.M.; Jain, J.; Shieh, S.R.; Wang, C.P.; Chuang, Y.C.; Liao, Y.F.; Zhang, D.Z.; Huang, T.; Lam, T.-N.; et al. Irreversible phase transformation in a CoCrFeMnNi high entropy alloy under hydrostatic compression. Mater. Today Commun. 2018, 14, 10-14. [CrossRef]

11. Huang, E.W.; Barabash, R.I.; Clausen, B.; Liaw, P.K. Cyclic-Loading Induced Lattice-Strain Asymmetry in Loading and Transverse Directions. Metall. Mater. Trans. A 2012, 43, 1454-1461. [CrossRef]

12. Huang, E.W.; Barabash, R.I.; Wang, Y.; Clausen, B.; Li, L.; Liaw, P.K.; Ice, G.E.; Ren, Y.; Choo, H.; Pike, L.M.; et al. Plastic behavior of a nickel-based alloy under monotonic-tension and low-cycle-fatigue loading. Int. J. Plast. 2008, 24, 1440-1456. [CrossRef]

13. Huang, E.W.; Barabash, R.I.; Clausen, B.; Liu, Y.L.; Kai, J.J.; Ice, G.E.; Woods, K.P.; Liaw, P.K. Fatigue-induced reversible/irreversible structural-transformations in a Ni-based superalloy. Int. J. Plast. 2010, 26, 1124-1137. [CrossRef]

14. Huang, E.; Clausen, B.; Wang, Y.; Choo, H.; Liaw, P.; Benson, M.; Pike, L.; Klarstrom, D. A neutron-diffraction study of the low-cycle fatigue behavior of HASTELLOY ${ }^{\circledR}$ C-22HSTM alloy. Int. J. Fatigue 2007, 29, 1812-1819. [CrossRef]

15. Huang, E.W.; Chang, C.K.; Liaw, P.K.; Suei, T.R. Fatigue induced deformation and thermodynamics evolution in a nano particle strengthened nickel base superalloy. Fatigue Fract Eng Mater Struct. 2016, 39, 675-685. [CrossRef]

16. Huang, E.W.; Barabash, R.I.; Ice, G.E.; Liu, W.; Liu, Y.L.; Kai, J.J.; Liaw, P.K. Cyclic-loading-induced accumulation of geometrically necessary dislocations near grain boundaries in an Ni-based superalloy. JOM: J. Miner., Met. Mater. Sci. 2009, 61, 53-58. [CrossRef]

17. Huang, E.W.; Barabash, R.; Jia, N.; Wang, Y.D.; Ice, G.E.; Clausen, B.; Horton, J.; Liaw, P.K. Slip-System-Related Dislocation Study from In-Situ Neutron Measurements. Metall. Mater. Trans. A 2008, 39, 3079. [CrossRef]

18. Huang, E.W.; Lin, C.M.; Juang, J.Y.; Chang, Y.J.; Chang, Y.W.; Wu, C.S.; Tsai, C.W.; Yeh, A.C.; Shieh, S.R.; Wang, C.P.; et al. Deviatoric deformation kinetics in high entropy alloy under hydrostatic compression. J. Alloys. Compd. 2019, 792, 116-121. [CrossRef]

19. Huang, E.W.; Yu, D.; Yeh, J.W.; Lee, C.; An, K.; Tu, S.Y. A study of lattice elasticity from low entropy metals to medium and high entropy alloys. Scr. Mater. 2015, 101, 32-35. [CrossRef]

20. Woo, W.; Huang, E.W.; Yeh, J.W.; Choo, H.; Lee, C.; Tu, S.Y. In-situ neutron diffraction studies on high-temperature deformation behavior in a CoCrFeMnNi high entropy alloy. Intermetallics 2015, 62, 1-6. [CrossRef] 
21. Von Dreele, R.B.; Larson, A.C. General Structure Analysis System (GSAS). Los Alamos Natl. Lab. Rep. LAUR 2004, 86-748.

22. Ribárik, G.; Ungár, T.; Ungár, J. MWP-fit a program for multiple whole-profile fitting of diffraction peak profiles by ab initio theoretical functions. J. Appl. Crystallogr. 2001, 34, 669-676. [CrossRef]

23. Velterop, L.; Delhez, R.; de Keijser, Th.H.; Mittemeijera, E.J.; Reefman, D. X-ray diffraction analysis of stacking and twin faults in f.c.c. metals. J. Appl. Cryst. 2000, 33, 296-306. [CrossRef]

24. Suzuki, Y.; Egami, T. Shear deformation of glassy metals: Breakdown of cauchy relationship and anelasticity. J. Non. Cryst. Solids 1985, 75, 361-366. [CrossRef]

25. Mughrabi, H. Dislocation clustering and long-range internal stresses in monotonically and cyclically deformed metal crystals. Rev. Phys. Appl. (Paris) 1988, 23, 367-379. [CrossRef]

26. Laplanche, G.; Kostka, A.; Horst, O.M.; Eggeler, G.; George, E.P. Microstructure evolution and critical stress for twinning in the CrMnFeCoNi high-entropy alloy. Acta Materialia 2016, 118, 152-163. [CrossRef]

27. Yu, P.F.; Cheng, H.; Zhang, L.J.; Zhang, H.; Ma, M.Z.; Li, G.; Liaw, P.K.; Liu, R.P. Nanotwin's formation and growth in an AlCoCuFeNi high-entropy alloy. Scr. Mater. 2016, 114, 31-34. [CrossRef]

28. Ungár, T. Microstructural parameters from X-ray diffraction peak broadening. Scr. Mater. 2004, 51, 777-781. [CrossRef]

29. Warren, B.E. X-ray diffraction. Phys. Today 1970, 23, 53. [CrossRef]

(C) 2019 by the authors. Licensee MDPI, Basel, Switzerland. This article is an open access article distributed under the terms and conditions of the Creative Commons Attribution (CC BY) license (http://creativecommons.org/licenses/by/4.0/). 